\title{
Assessment of Nurses' Knowledge and Practice for Prevention of Infection in Burn Patients
}

\author{
Nazia Ahmad Buksh ${ }^{1 *}$, Mansoor Ghani ${ }^{2}$, Shahnaz Amir ${ }^{3}$, Kainat Asmat ${ }^{4}$, Samra Ashraf ${ }^{5}$ \\ ${ }^{1,3,4,5}$ Student, University of Health Sciences Lahore, Pakistan \\ ${ }^{2}$ Head of Department, Institute of nursing, University of Health Sciences Lahore, Pakistan
}

DOI: $10.36348 /$ SJMPS.2019.v05i10.005

| Received: 29.08.2019 | Accepted: 05.09.2019| Published: 20.10.2019

*Corresponding author: Nazia Ahmad Buksh

\section{Abstract}

Objective: To assess nurses' knowledge and evaluate their practice for prevention of infection among burn patients, also to suggest guidelines especially of nursing practice for prevention of infection in burn patients. Study Design: A descriptive cross sectional study. Place and Duration: Six months, from April 2017 to Sep 2017, in burn units of 03 selected hospitals of Lahore. Methods: The study included all nurses who were providing care to burn patients (N=48). Nurses' knowledge was assessed by using a self-structured questionnaire and their practices were evaluated by direct monitoring using an observational Checklist. Results: Females constituted $100 \%$ of study participants. $62.5 \%$ of nurses had a GNM whilst $37.5 \%$ had a Nursing degree. $73 \%$ (35) of participants had less than 2 years of experience working in the Burns Unit. Knowledge regarding infection control was received by $89 \%$ of nurses but their source of information was practice not in-service educational programs. Consequently they had unsatisfactory level of knowledge and practices indicated by knowledge and practice score less than 75\%. Conclusion: The findings of the current study revealed nurses' low level of knowledge and practices. Hence, healthcare settings are required to organize advanced training sessions and to develop unit specific clinical guidelines and protocols.

Keywords: Burn, Nurses, infection control, Knowledge, Practice.

Copyright @ 2019: This is an open-access article distributed under the terms of the Creative Commons Attribution license which permits unrestricted use, distribution, and reproduction in any medium for non-commercial use (NonCommercial, or CC-BY-NC) provided the original author and source are credited.

\section{INTRODUCTION}

Burn injuries are among the most distressful trauma and a considerable public health issue all over the world [1]. Approximately 180,000 deaths occur by burn every year estimated by world health organization (WHO) in 2018. These burn injuries extensively occur in low income and middle income countries, in the same way death rate is higher in low income countries as compared to high income countries. Burns could also leading to many disability-adjusted life-years (DALYs) lost in low and middle-income countries [2]. WHO has reported much higher incidence in Pakistan approximately $1388 / 100000$ annually as compared to global incidence of 110/100000 per annum [3].

Treatment for burn patients depends on the cause, depth, and surface area of burn injury. Treatment on basis of depth of burn includes many different approaches such as wound debridement, application of the modern hydrocolloid; silicone dressings and chlorhexidine coated conventional dressings for superficial burn. However, deep dermal burn treatment protocol involve different dressings which stimulate epithelialization and many grafting procedures[4] Furthermore, Empiric Antimicrobial therapy is not recommended to treat fever because most of the time these patients have fever just because of the systemic inflammatory response to burn[5].

The most common complications associated with burn are burn wound infections while other frequent complications are sepsis, pneumonia, lung failure, acute renal failure, acute respiratory distress syndrome (ARDS), and multi organ failure[6]. Most of burn wound infections eventually cause morbidity and mortality of burn patients and become very challenging matter for burn team [5]. It was also estimated that about $75 \%$ of the mortality among burn patients related to sepsis which is dangerous body response to infection[7].

Burn wound infections can spread like other infections and follow the universal chain of infection that includes three components, causative agents of infection; ways of transmission; and patient susceptibility for infection. Almost all types of pathogens found in burn patients such as gram positive 
and negative and fungal organisms. The typical burn wound is primarily contaminated with gram-positive organisms, which mostly replaced by gram- negative more resistant organism after a week. Secondly, transmission of infection. In the case of burn patients, it majorly by direct or indirect contact. This contact can usually occur either through the hands of the nurse or by any other personnel taking care for the patient or during use of equipment not properly decontaminated. Finally, susceptibility of the patient. In burn patients, damage to physical defense can lead to contamination of wound by opportunistic and pathogenic organisms[5].

The Centre for Disease Control and Prevention and the Society for Health Care Epidemiology present general infection-control guidelines for health settings. However, no one currently recommended infection control guidelines specifically in burn unit. Until further evidence based data are available we have to rely on above mentioned general measures of infectioncontrol[8].

A European study provided strong evidence regarding contribution of infection control recommendations in lowering MRSA prevalence rates. They reported in the form of mean difference of MRSA prevalence rate (i) $10.3 \%$ with the use of alcohol-based sanitizers for hand hygiene and $11.2 \%$ by placing MRSA patients in single isolated rooms. They also communicated that failure to implement isolation policies would higher resistance levels with the mean difference up to $12 \%$ [9].

Another technique that provides evidence to reduce pathogen load and prevention of hospital acquired infection is bath of burn patients with chlorhexidine solution. Twice-daily chlorhexidine gluconate $(\mathrm{CHG})$ bath protocol is very effective for lowering bioload of organismsand[10].

The Centre for Disease Control and Prevention and the Society for Health Care Epidemiology present general infection-control guidelines for health settings. However, no one currently recommended infection control guidelines specifically in burn unit. Until further evidence based data are available we have to rely on above mentioned general measures of infectioncontrol[8].

A descriptive study conducted for assessment of knowledge and practice of nurses for infection control in burn patients. It showed that most of the studied sample (90\%) had satisfactory level of knowledge $(\geq 75 \%)$ and unsatisfactory level of practice $(<85 \%)$ regarding infection control measures[11].

In this regards, application of infection control measures in burn unit can be uphill battle and strenuous experience. It can be stressful because Nurses need to follow multiple protocols in caring for the patients. It also provoking because nurses play a vital role during bio-psychosocial assessment and management of their patients, it may also be rewarding because nurses can demonstrate their understanding of holistic nursing care[12].

Burn injuries are potentially life threatening conditions and burn patients demand exceptional care. This care should be given by knowledgeable and competent nurses following specific guideline or protocols that will reduce the occurrence of infection and complications. Hence, there is evidence that management and care of patient with burn injury need a unique body of knowledge and skills from a responsible multidisciplinary team members especially the nurse, and specific infection control guidelines should be develop for preventing infections especially hospital acquired infection (HAI), HAIs are considered an undesirable outcome, indicator of the quality of patient care lead to severe complications.

\section{METHODOLOGY \\ Study Design \\ Descriptive Cross-sectional design}

\section{Setting}

The study was conducted in burn units of 03 selected hospitals of Lahore, Punjab Region including Jinnah Burn \& Reconstructive Surgery Centre Lahore, Mayo Hospital Lahore and Shalimar hospital Lahore.

\section{Subjects}

The study included all nurses who were providing care to burn patients $(\mathrm{N}=48)$.

\section{Inclusion Criteria}

All registered nurses working in burn units for at least six months before the start of data collection were considered eligible.

\section{Exclusion Criteria}

Nurses working in burn care units, who were not involved in direct patient care e.g. Head nurses, Clinical Instructors and Nurse Managers and nurses who have worked for less than six months in the Burns Unit.

\section{Ethical Considerations}

Ethical approval was taken from Ethical Review Committee of University of Health Sciences Lahore, Pakistan. Written permission was obtained from deans/medical directors of respective study hospitals. Written consent describing the purpose and benefits of the study was taken from study participants. 


\section{Tools of Data Collection}

Two data collection instruments were designed and utilized to collect data pertinent to the current study.

\section{Tool I: Questionnaire on Nurses' knowledge of prevention of Infections in burn patients.}

The study objectives and literature review guided the design of the questionnaire. It was mainly divided into two parts: Part I consists of Demographic characteristics such as gender, age, education, years of work experience, attendance of training about preventing Infections in burn patients, and availability of protocol regarding Infection prevention in burn unit. Part II consists of 30 items on Knowledge for prevention of Infections. Items were constructed under 03 main domains including knowledge of burn \& its associated infections (Question 1 to 8), microorganisms of burn wound (Question 9 to 11), burn wound culture (Question 12 to 14), infection control in burn unit (Question 15 to 20) and infection control during care of burn patients (Question 21 to 30).Each question with 04 answer choices (01 correct and 03 incorrect). Each question was scored 01 for a correct and 0 for an incorrect answer. Aggregate knowledge scores range from $0-30$. Scores of less than $75 \%$ were considered unsatisfactory level of knowledge; however scores of $75 \%-100 \%$ were considered satisfactory level of knowledge.

\section{Tool II: Performance Observational Checklist:}

This tool was designed after thorough review of relevant literature. The tool was intended to observe nurses' practices while caring for burn patients in burn unit. It covered 02 main infection control practices including observation of standard precautions (observation 1) with 15 sub-items and specific infection control measures (observation 2) with 11 sub-items. Each nurses was observed by researcher three times and each nurse's performance was recorded as correctly done or not done/ incompletely done/incorrectly done. The main purpose of this observation is that each nurse know or not know about infection prevention practices. If study participant know about procedure, he/she is allowed to perform otherwise perform incorrectly or not performing that practices. 01 score was allocated to two or three times correct performance in three times observations, and 0 to not done/incompletely done/incorrectly done/one time performance. Total practice scores range from 0-26. Scores less than $75 \%$ were considered unsatisfactory practice while scores from $75 \%-100 \%$ were considered satisfactory practice.
Validity and reliability of Data Collection Tools

In current study, internal consistency reliability of self-constructed questionnaire was tested through pilot study by applying Cronbach's Alpha through SPSS IBM version 20. It was calculated as with internal consistency of 0.685 which indicated that the instrument was reliable for data collection.

\section{Pilot Study}

Before embarking on the full study, selfconstructed questionnaire was pre-tested. A pilot study was carried out on 06 staff nurses that were $12 \%$ of sample size to test the feasibility of study design, impartiality and applicability of data collection tool. Participants were selected according to their voluntary consent on basis of non-probability sampling technique. Selected participants were not included in actual study.

\section{Data Collection Procedure}

After obtaining official permissions, data were collected over a period of 06 months starting from Apr 2017 to Sep 2017. The selected burn units were visited on daily basis and nurses were approached during morning, evening and night shifts. After detailed information regarding purpose and benefits of the study, a written consent was taken from all the nurses. Then the nurses were submitted with 1st data collection tool; Questionnaire. Proper guidance was provided for understanding to the questions asked. Nurses were requested to answer as per their own understanding to the question. It took 25-30 minutes in average for each nurse to fill in the questionnaire. Participant nurses' direct observation was carried out utilizing 2nd data collection tool; Observational Check list. Each nurse was directly observed while she was performing Care for three different times to exclude the subjectivity. Obtained data were converted into numeric form, and the average of two observations was calculated.

\section{Data Analysis}

The response rate was $100 \%$. Data was processed and analysed through SPSS software version IBM 20. Descriptive statistics (mean, standard deviation, frequencies and percentages) were used to describe the demographic and practice characteristics of study participants and other important variables such as total knowledge and practice scores.

\section{RESULTS}

SECTION 1: Table 1 describes demographic and practice characteristics of participant nurses which include gender, age, qualification, experience in burn units, training received on prevention and availability of protocol at respective unit of study. 
Nazia Ahmad Buksh et al; Saudi J Med Pharm Sci, Oct 2019; 5(10): 846-855

Table-1: Demographic and Practice Characteristics of Studied Sample (N=48)

\begin{tabular}{|l|l|l|}
\hline \multicolumn{1}{|c|}{ Frequency } & Percentage \\
\hline Gender & & \\
\hline Female & 48 & 100.0 \\
\hline Age & & \\
\hline $21-30$ & & \\
\hline $31-40$ & 27 & 56.2 \\
\hline 41 or above & 19 & 39.6 \\
\hline Total & 2 & 4.2 \\
\hline Qualification & 48 & 100.0 \\
\hline General Nursing and Midwifery(GNM) & & \\
\hline & 30 & 62.5 \\
\hline Bachelor of science in nursing(BSN) & 18 & 37.5 \\
\hline Total & & \\
\hline & 48 & 100.0 \\
\hline Experience (Burn Unit) & & \\
\hline$<1$ YEAR & & \\
\hline $1-2$ YEARS & 18 & 37.5 \\
\hline $3-5$ YEARS & 17 & 35.4 \\
\hline $5+$ YEARS & 12 & 25.0 \\
\hline Total & 1 & 2.1 \\
\hline & 48 & 100.0 \\
\hline INFORMATION Received regarding infection control & & \\
\hline YES & 43 & 89.6 \\
\hline NO & 5 & 10.4 \\
\hline Total & 48 & 100.0 \\
\hline & & \\
\hline PROTOCOL availability regarding infection control & & \\
\hline YES & 11 & 22.9 \\
\hline NO & 37 & 77.1 \\
\hline Total & 48 & 100.0 \\
\hline & & \\
\hline & & \\
\hline & & \\
\hline
\end{tabular}

\section{SECTION II}

Table 2 illustrates frequency distribution of Studied Sample according to their knowledge on each item of SelfAdministered Questionnaire 
Nazia Ahmad Buksh et al; Saudi J Med Pharm Sci, Oct 2019; 5(10): 846-855

Table-2: Frequency Distribution of Studied Sample as regards to Knowledge Scores ( $N=48$ )

\begin{tabular}{|c|c|c|}
\hline Items on Questionnaire & $\begin{array}{l}\text { Correct } \\
\quad \text { f }\end{array}$ & $\begin{array}{c}\text { Correct } \\
\%\end{array}$ \\
\hline 1. Burn patients are unique due to & 27 & 56.2 \\
\hline 2. According to American burn association which patient should be referred to burn unit & 20 & 41.7 \\
\hline 3.Which zone is located in the center of burn wound & 9 & 18.8 \\
\hline 4. Superficial burn treatment protocol includes & 36 & 75.0 \\
\hline 5. One of major burn infection is burn wound cellulitis: which is & 21 & 43.8 \\
\hline 6. Common cause of fever in burn patients & 20 & 41.7 \\
\hline 7. What is basic cause of burn wound infection & 4 & 8.3 \\
\hline 8. Sepsis syndrome clinically manifested by following & 44 & 91.7 \\
\hline 9. Burn wounds initially colonized with & 16 & 33.3 \\
\hline 10. Which microorganisms are more resistant in burn patients? & 14 & 29.2 \\
\hline 11. The principle causative agent of burn cellulitis & 30 & 62.5 \\
\hline 12. When routine surveillance cultures should be taken & 13 & 27.1 \\
\hline 13. Recommendations about wound cultures for large burn injuries & 26 & 54.2 \\
\hline 14. Semi Quantitative swab culture provide information about & 11 & 22.9 \\
\hline \multicolumn{3}{|l|}{ D. Infection control in burn unit } \\
\hline 15. Which is more effective method for prevention of infection in isolation room & 6 & 12.5 \\
\hline $\begin{array}{l}\text { 16. Which item/s are not allowed in burn unit because they harbor resistant specie (Gram negative) of } \\
\text { microorganism }\end{array}$ & 37 & 77.1 \\
\hline $\begin{array}{l}\text { 17. According to Spaulding classification of medical devices, which item require high level of } \\
\text { disinfection as semi critical item }\end{array}$ & 31 & 64.6 \\
\hline $\begin{array}{l}\text { 18. According to CDC guidelines of disinfection, Immersion time of equipment for high level } \\
\text { disinfection (HLD) with } 2.4 \% \text { glutaraldehyde is }\end{array}$ & 9 & 18.8 \\
\hline 19. Contact time for surface disinfection is & 14 & 29.2 \\
\hline 20. High touch surface areas must be clean and disinfect & 28 & 58.3 \\
\hline \multicolumn{3}{|l|}{ E. Infection Control during care of the patient } \\
\hline $\begin{array}{l}\text { 21. How many times chlorhexidine bath recommended for burn patients for prevention of infection in } \\
\text { burn patients }\end{array}$ & 14 & 29.2 \\
\hline 22.Chlorhexidine bath in burn patients & 38 & 79.2 \\
\hline 23. Which factor leads to acquisition of antibiotic resistant organism in burn patient's & 22 & 45.8 \\
\hline 24. What is important for preparation of an isolation room & 37 & 77.1 \\
\hline 25. Which precautions are most important for prevention of MRSA in burn patients? & 23 & 47.9 \\
\hline 26. Patients require additional infection control precautions & 20 & 41.7 \\
\hline 27. Nasal decolonization of MRSA patients done by & 14 & 29.2 \\
\hline 28. Aquatic environment of hydrotherapy room is difficult to decontaminate because & 4 & 8.3 \\
\hline 29.Specific antiseptic recommended for hand washing & 22 & 45.8 \\
\hline 30. Important step during removal of personal protective equipment & 34 & 70.8 \\
\hline
\end{tabular}

With regard to the knowledge of burn and its infections, more than half 27 (56\%) nurses knew that burn patients are unique due to propensity to disperse microbes in environment and only $20(41 \%)$ of them knew about referral criteria of American Burn Association. Fever is very common in burn patients but its cause is unique, only $4(8 \%)$ nurses knew that common cause of fever in burn patients is systemic inflammatory response not a pathogenic action of microorganisms.

With reference to knowledge for accumulation of microorganisms in burn wounds, $16(33 \%)$ of them knew that burn wounds initially colonized gram positive organisms and only $14(29 \%)$ of the nurses agreed that exogenous microorganism are more resistant as compared to endogenous. Burn wound cellulitis is most common infection in burn patients, 30 $(62 \%)$ nurses knew its causative agent.
With reference to knowledge regarding infection control measures especially in burn unit, majority $37(77 \%)$ nurses mentioned plants and flowers harbor resistant organisms that's why these are not allowed in burn unit. Recent researches find out that chlorhexidine bath in burn patients lead to massive decrease in HAIs but findings of present study represent that only $14(29 \%)$ nurses knew about chlorhexidine bath and its suggested frequency in burn patients. In addition, nearly half of nurses $23(47 \%)$ agreed that contact precautions are most effective than other precautions. Where basic cause of ineffective decontamination of hydrotherapy room was identified by only $4(8 \%)$ nurses.

Fig 1 presents the percentage distribution of studied sample according to their knowledge for preventing Infections among burn patients. It clarifies that $46(95 \%)$ out of 48 nurses have Unsatisfactory level of knowledge $(<75 \%)$, However $2(4 \%)$ nurses have Satisfactory level of knowledge (75\% - 100\%). 


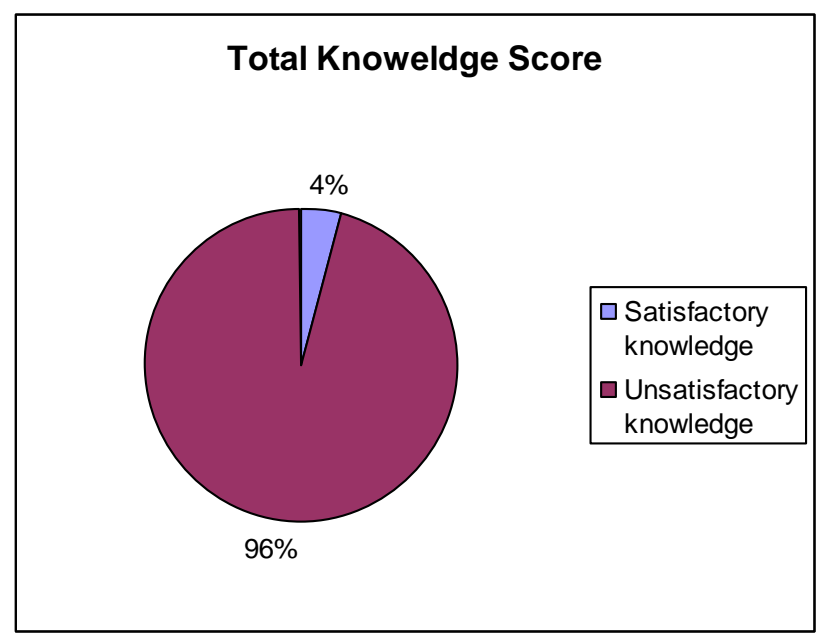

Fig-1: Total Knowledge

\section{SECTION III}

\section{Analysis of Performance Observational Checklist}

Table-3: Frequency distribution of study sample according to their performance regarding infection control in burn unit.

\begin{tabular}{|c|c|c|c|c|}
\hline \multirow[t]{2}{*}{ Observation 1 (Standard Precaution) } & \multicolumn{2}{|c|}{$\begin{array}{l}\text { No / incorrect /one time } \\
\text { performance" }\end{array}$} & \multicolumn{2}{|c|}{$\begin{array}{l}\text { two times/three times } \\
\text { correct performance }\end{array}$} \\
\hline & $F$ & $\%$ & $F$ & $\%$ \\
\hline $\begin{array}{l}\text { Hand washing with alcohol rub / } \\
\text { antimicrobial material before the procedure }\end{array}$ & 36 & 75.0 & 12 & 25.0 \\
\hline Mask & 12 & 25.0 & 36 & 75.0 \\
\hline Gown & 41 & 85.4 & 7 & 14.6 \\
\hline Gloves & 8 & 16.7 & 40 & 83.3 \\
\hline Use No Touch Technique & 48 & 100.0 & 0 & 0 \\
\hline $\begin{array}{l}\text { Proper Disinfection of Medical Devices } \\
\text { Thermometer }\end{array}$ & 48 & 100.0 & 0 & 0 \\
\hline Sphygmomanometer & 48 & 100.0 & 0 & 0 \\
\hline Stethoscope & 48 & 100.0 & 0 & 0 \\
\hline Blood glucose monitoring equipment & 47 & 97.9 & 1 & 2.1 \\
\hline Safe Disposal of Needle and Sharp materials & 28 & 58.3 & 20 & 41.7 \\
\hline Proper Handling of Soiled linen & 40 & 83.3 & 8 & 16.7 \\
\hline $\begin{array}{l}\text { Proper Disposal of Personal Protective } \\
\text { Equipment's }\end{array}$ & 35 & 72.9 & 13 & 27.1 \\
\hline Proper Disposal of Clinical Waste & 31 & 64.6 & 17 & 35.4 \\
\hline Hand washing after the procedure & 34 & 70.8 & 14 & 29.2 \\
\hline Obeying Patient visitor policy & 29 & 60.4 & 19 & 39.6 \\
\hline \multicolumn{5}{|l|}{ Observation 2 ( specific Precautions) } \\
\hline Hand Washing before procedure & 38 & 79.2 & 10 & 20.8 \\
\hline $\begin{array}{l}\text { Prepare clean skin with an antiseptic before } \\
\text { peripheral venous catheter insertion }\end{array}$ & 16 & 33.3 & 16 & 33.3 \\
\hline $\begin{array}{l}\text { Antiseptics should be allowed to dry prior to } \\
\text { placing the catheter }\end{array}$ & 46 & 95.8 & 2 & 4.2 \\
\hline $\begin{array}{l}\text { Application of transparent \& semi-permeable } \\
\text { dressing }\end{array}$ & 14 & 29.2 & 34 & 70.8 \\
\hline Aseptic measures during dressing & 41 & 85.4 & 7 & 14.6 \\
\hline Horizontal surfaces & 20 & 41.7 & 28 & 58.3 \\
\hline Walls & 32 & 66.7 & 16 & 33.3 \\
\hline Surfaces of furniture/Bed & 38 & 79.2 & 10 & 20.8 \\
\hline Monitor & 39 & 81.2 & 9 & 18.8 \\
\hline Chlorhexidine bath & 47 & 97.9 & 0 & 0 \\
\hline $\begin{array}{l}\text { Fumigation of cubicle after discharging of the burn } \\
\text { patient }\end{array}$ & 28 & 58.3 & 20 & 41.7 \\
\hline
\end{tabular}

Frequency Distribution of Studied Sample as regards to Performance Scores $(\mathrm{N}=50)$ 
With regard to standard precautions, only $25 \%$ and $29 \%$ among the nurses washed hands prior and after the various procedures that demand hand washing. On the other hand, most of the nurses wore gloves $(83 \%)$ and masks $(75 \%)$ but few $14 \%$ wore gowns. No one among nurses followed no touch technique and disinfection of various medical devices. With reference to specific precautions, only 2 nurses out of 48 allow to dry antiseptic before intravenous access. Only 7 (14\%) nurses follow aseptic measures during dressing of burn patients. No one among nurses followed the protocol of chlorhexidine bath.

Fig 2 demonstrates percentage distribution of study sample according to their performance scores. It indicates that $0(0 \%)$ out of 48 nurses have satisfactory level of performance (75\%-100\%), however 48 (100\%) out of 48 nurses have unsatisfactory performance level $(<75 \%)$.

\section{Fig 2 Total Performance}

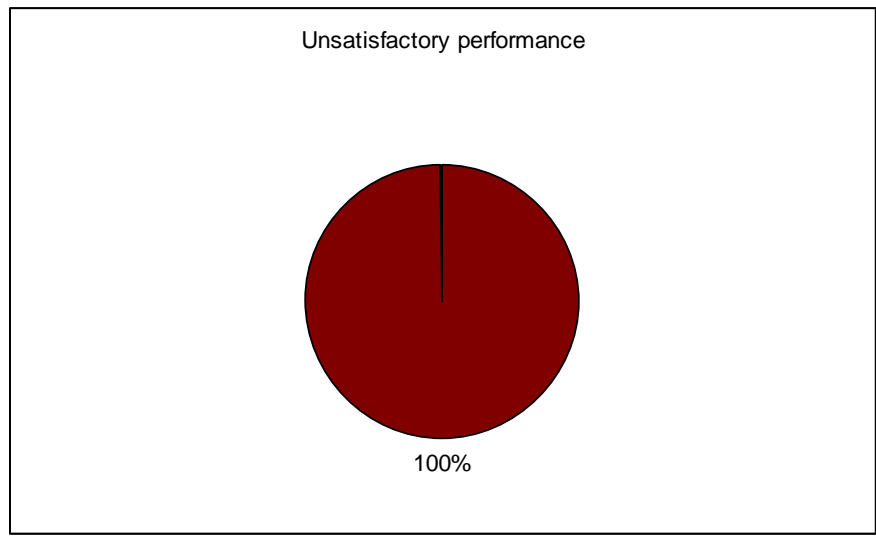

Fig-2: Percentage Distribution of studied sample according to total Performance Scores

\section{DISCUSSION}

The findings emerged from the data are that nurses in study areas have insufficient knowledge and practice about infection control in burn patients. However, they received information through practice but in-service education programs need to develop to prepare nurses for prevention of infection.

Nurses' knowledge of infection control has fundamental role in preventing hospital acquired infections among burn patients. As majority of study participants received information regarding prevention of infection, the finding of current study showed low level of knowledge among nurses as indicated by the low percentages of accurate responses. The finding showed that $96 \% \quad(n=48)$ has unsatisfactory level of knowledge indicated by satisfactory knowledge score $75 \%-100 \%$. These findings are comparable to Atalla $e t$ $a l$. work on compliance of nurses with infection control polices concluded that participants had low-level regarding infection control guidelines [13]. In contrast nurses' knowledge about guidelines for preventing burn related infections was adequate $(90 \%)$ but in contrary knowledge of participants regarding burn (definition. causes \& calculation of burn area) and nosocomial infection in burn unit was low[11].

It is recommended that patients with larger burn injuries be isolated in private rooms to prevent cross contamination. It was observed that laminar airflow is the most effective strategy to prevent cross infection [5]. Likewise, twice a day chlorhexidine bath protocol is most effective method recommended for burn patients for decreasing hospital acquired infections even up to zero [10]. Findings of current study showed very low knowledge regarding this protocol because of unavailability of in-service education regarding prevention of HAI in burn patients. The present study revealed that majority $(88 \%)$ of nurses lack knowledge about these procedures. The findings are in agreement with Mohammadzadeh et al. revealed poor knowledge of health care workers regarding infection control precautions [14]. The findings are contrary to Sorte, who reported that nurses have good $(76 \%)$ knowledge regarding prevention of hospital acquired infection [15].

Burn patients with resistant organisms (for example MRSA) may serve as reservoir for transmission of infection to other patients, so these patient requires various precautions specially contact precautions [5]. The present study revealed low knowledge for such precautions especially contact precautions which are more essential for MRSA patients. The fining is consistent with Askarian et al. demonstrated low knowledge of health care workers for standard precautions and also reported that $90 \%$ of participants need extra infection control education [16].

Nurses' practices complying with evidence based guidelines about burn care have a crucial impact on preventing Infections among burn patients. The present study illustrates that not a single nurse has 
satisfactory level of practice as indicated by total performance scores $75 \%-100 \%$. These findings may involve many factors such as low nurse to patient ratio, few burn centres, less experience in burn centre, unavailability of in-service training courses, diminished institutional resources and absence of specific protocol etc. Nurses' practices complying with evidence based guidelines about burn care has crucial impact on preventing Infections among burn patients. All of the nurses showed unsatisfactory level of practice as indicated by total performance scores $<75 \%$. This finding is supported by study conducted by El-Sayed $e t$ al. in burn centre of Egypt who reported very low percentage of study sample had satisfactory level of practice for preventing infection in burn patients [11].

As nurses' practices for infection control in burn patients mainly involve standard precautions but findings of present study showed very low performance. This may be due to overburden of nurses and unavailability of resources. These consequences are also shared by Abdulraheem et al. who observed poor compliance of health care workers regarding standard precautions during care of patients [17].

Hand hygiene is the first initial step towards successful infection control in any healthcare setup including burn Centre. Although the results found that all of nurses had unsatisfactory level of practice but regarding hand washing $25 \%$ to $29 \%$ nurses shows compliance to hand hygiene. Many research studies investigating the compliance to hand hygiene such as Karaaslan et al. also observed only $41 \%$ compliance similar to findings of present study[18].

Use of Personal protective equipment (PPE) includes the use of mask, sterile gown and sterile gloves during care of patient. Carrer et al. found reduction in the risk of skin colonization when PPE were implemented [19]. In present study nurses shows satisfactory compliance for use of masks $(75 \%)$, gloves (83\%) but poor compliance for use of gowns (14\%) because they thought that gowns are not necessary during care of patient. Consequently an overall low performance for PPE was noted because even not wearing gowns can lead to cross contamination between the patients. Findings are agreement with Ganczak and Szych, who reported only low (5\%) compliance to PPE by nurses [20].

Cleaning and disinfection of medical devices is so important to prevent buildup of various microorganisms onto medical devices, it is highly likely that bacteria will grow if left unchecked or without disinfection properly [21]. Findings of present study showed very low compliance with it. In consistent with Quinn et al. who concluded with that equipment cleaning was observed on approximately one-fourth of the person days for registered nurses ( only 23\%)[22].

\section{CONCLUSION}

Based on the findings of the study, it is concluded that nurses working in burn units of 03 hospitals of Lahore, Punjab region have low knowledge and practices regarding prevention of Infections among burn patients. Therefore, hospitals are required to organize adequate trainings and to develop unit specific clinical infection control guidelines and protocols.

\section{Suggested Nursing Guidelines for burn patients}

Based on the study findings and pre-mentioned discussion, the present study recommends following guidelines:-

\section{Isolation Guidelines}

- Standard precautions should be applied when care is provided to all patients with burn injury.

- Appropriate barrier garb (clean gowns, gloves \& plastic aprons) is recommended for any burn patient during contact.

- Standard precautions also include appropriate hand washing.

- Proper removal of PPE after providing care to patients.

- Gloves should be change when they contaminated with body fluids of the patient and before starting another nursing procedure.

- Use sterile equipment along with wearing gloves, caps and masks when dealing with open burn wounds or other invasive procedures.

- Appropriate decontamination of equipment and surfaces should be before storage or use on other patients and proper barrier grab should also worn during decontamination of equipment.

\section{Need Specific Precautions}

- Specific group of burn patients are distinctive and demand extra precautions such as patients with larger burn injury greater than $25 \%$ to $30 \%$ TBSA and those identified with multi resistant organisms (MDR).

- These patients should be placed in isolated cabins or other enclosed areas to ensure physical separation because their contaminated wounds becomes a huge source for transmission of organisms by spreading them in the surrounding environment.

\section{Environmental Issues}

- Surrounding environment can be source of resistant organisms so prohibited items should not allowed in burn units such as flowers and pets.

\section{Sites of Infection and Prevention Techniques Burn Wound Infection}

- Regular assessment of burn wound help in early identification of deterioration in the character, odour or amount of wound drainage. 
- $\quad$ Strict aseptic technique should be applied during dressing of open wounds of burn.

- Twice a daily chlorhexidine bath is recomented in burn patients

- If necrotic tissue develops over wounds, a debriding dressing should be applied.

\section{Prevent Intravenous infection}

- Personal protective equipment should be used correctly and select site away from wound.

- Maintained hand hygiene before and after the procedure.

- Cleaned the skin at the site of entry with antiseptic swab and used a circular motion, moving from the centre outward

- Permitted solution to dry on the skin

- Used a no-touch technique for any part of the needle or cannula.

- Checked any leakage or damage or contaminated before insertion.

- Apply transparent dressing after insertion.

- Label the dressing with the date and time of insertion.

\section{ACKNOWLEDGEMENT}

The authors would like to express their earnest gratefulness to Medical Superintendents and burn nurses of study hospitals for their co-operation. The authors also acknowledge the expertise of University of Health Sciences medical and nursing faculty for their valuable guidance and support.

\section{REFERENCES}

1. Qader, A. R., \& Muhamad, J. A. (2010). Nosocomial infection in sulaimani burn hospital, IRAQ. Annals of burns and fire disasters, 23(4), 177.

2. WHO. (2018). Burns.

3. Othman, N., \& Kendrick, D. (2010). Epidemiology of burn injuries in the East Mediterranean Region: a systematic review. BMC public health, 10(1), 83 .

4. Leseva, M., Arguirova, M., Nashev, D., Zamfirova, E., \& Hadzhyiski, O. (2013). Nosocomial infections in burn patients: etiology, antimicrobial resistance, means to control. Annals of burns and fire disasters, 26(1), 5.

5. Weber, J., McManus, A., \& Nursing Committee of the International Society for Burn Injuries. (2004). Infection control in burn patients. Burns, 30(8), A16-A24.

6. Spanholtz, T. A., Theodorou, P., Amini, P., \& Spilker, G. (2009). Severe burn injuries: acute and long-term treatment. Deutsches Ärzteblatt International, 106(38), 607.

7. Donati, L., Scamazzo, F., Gervasoni, M., Magliano, A., Stankov, B., \& Fraschini, F. (1993). Infection and antibiotic therapy in 4000 burned patients treated in Milan, Italy, between 1976 and 1988. Burns, 19(4), 345-348.

8. Laurie, C. D., Saffle, J. R., Chung, K. K., \& Cancio, L. C. (2008). Prevention and management of infections associated with burns in the combat casualty. Journal of Trauma and Acute Care Surgery, 64(3), S277-S286.

9. Mackenzie, F. M., Bruce, J., Struelens, M. J., Goossens, H., Mollison, J., Gould, I. M., \& ARPAC Steering Group. (2007). Antimicrobial drug use and infection control practices associated with the prevalence of methicillin-resistant Staphylococcus aureus in European hospitals. Clinical microbiology and infection, 13(3), 269-276.

10. Popp, J. A., Layon, A. J., Nappo, R., Richards, W. T., \& Mozingo, D. W. (2014). Hospital-acquired infections and thermally injured patients: chlorhexidine gluconate baths work. American journal of infection control, 42(2), 129-132.

11. El-Sayed, M., Gomaa, M., \& Abdel-Aziz, M. (2015). Nurses' knowledge and practice for prevention of infection in burn unit at a university hospital: suggested nursing guidelines. $J$ Nurs Health Sci, 4(4), 62-9.

12. Greenfield, E. (2010). The pivotal role of nursing personnel in burn care. Indian journal of plastic surgery: official publication of the Association of Plastic Surgeons of India, 43(Suppl), S94.

13. Greenfield, E. (2010). The pivotal role of nursing personnel in burn care. Indian journal of plastic surgery: official publication of the Association of Plastic Surgeons of India, 43(Suppl), S94.

14. Mohammadzadeh, M., Behnaz, F., \& Parsa, S. (2013). Knowledge, practice and attitude towards standard isolation precautions in nurses, auxiliary nurses and midwives of Shahid Sadoughi hospital Yazd, Iran. Int J Infect Control, 9(1).

15. Sorte, D. (2015). Nurses Knowledge Related to Prevention of Nosocomial Infection (Vol. 4).

16. Askarian, M., Memish, Z. A., \& Khan, A. A. (2007). Knowledge, practice, and attitude among Iranian nurses, midwives, and students regarding standard isolation precautions. Infection Control \& Hospital Epidemiology, 28(2), 241-244.

17. Abdulraheem, I. S., Amodu, M. O., Saka, M. J., Bolarinwa, O. A., \& Uthman, M. M. B. (2012). Knowledge, awareness and compliance with standard precautions among health workers in north eastearn Nigeria. J Community Med Health Edu, 2(3), 1-5.

18. Karaaslan, A., Kepenekli Kadayifci, E., Atıcı, S., Sili, U., Soysal, A., Çulha, G., ... \& Bakır, M. (2014). Compliance of healthcare workers with hand hygiene practices in neonatal and pediatric intensive care units: overt observation. Interdisciplinary perspectives on infectious diseases, 2014. 
19. Carrer, S., Bocchi, A., Bortolotti, M., Braga, N., Gilli, G., Candini, M., \& Tartari, S. (2005). Effect of different sterile barrier precautions and central venous catheter dressing on the skin colonization around the insertion site. Minerva Anestesiol, 71(5), 197-206.

20. Ganczak, M., \& Szych, Z. (2007). Surgical nurses and compliance with personal protective equipment. Journal of Hospital Infection, 66(4), 346-351.

21. Quinn, M. M., Henneberger, P. K., Braun, B., Delclos, G. L., Fagan, K., Huang, V., \& Maher, K.
A. (2015). Cleaning and disinfecting environmental surfaces in health care: toward an integrated framework for infection and occupational illness prevention. American journal of infection control, 43(5), 424-434.

22. Quinn, M. M., Henneberger, P. K., Braun, B., Delclos, G. L., Fagan, K., Huang, V., \& Maher, K. A. (2015). Cleaning and disinfecting environmental surfaces in health care: toward an integrated framework for infection and occupational illness prevention. American journal of infection control, 43(5), 424-434. 